\title{
A FIXED POINT THEOREM, A PERTURBED DIFFERENTIAL EQUATION, AND A MULTIVARIABLE VOLTERRA INTEGRAL EQUATION
}

BY

\section{DAVID LOWELL LOVELADY}

ABSTRACT. A fixed point theorem is obtained for an equation of the form $u=T[p, f+G[u]]$. This theorem is then applied to a functionally perturbed ordinary differential equation of the form $u^{\prime}(t)=f(t)+A(t, u(t))+G[u](t) ; u(0)=p$, and, as a consequence of this, Fredholm integrodifferential equations of the form $z^{\prime}(t)=f(t)+\phi(t, z(t))+\int_{0}^{\infty} a(t, s) \omega(z(s)) d s, z(0)=p$. Applications are also made to a multivariable Volterra integral system

$$
\begin{aligned}
& u_{1}(s, t)=g_{1}(s, t)+\int_{0}^{s} \int_{0}^{t} F\left[u_{1}(x, y), u_{2}(x, y), u_{3}(x, y)\right] d y d x \\
& u_{2}(s, t)=g_{2}(s, t)+\int_{0}^{t} F\left[u_{1}(s, y), u_{2}(s, y), u_{3}(s, y)\right] d y \\
& u_{3}(s, t)=g_{3}(s, t)+\int_{0}^{s} F\left[u_{1}(x, t), u_{2}(x, t), u_{3}(x, t)\right] d x,
\end{aligned}
$$

and, as a corollary to this, a differential equation of the form

$$
\begin{aligned}
& \frac{\partial^{2}}{\partial s \partial t} u(s, t)=f(s, t)+F\left[u(s, t), \frac{\partial}{\partial s} u(s, t), \frac{\partial}{\partial t} u(s, t)\right], \\
& u(s, 0)=\phi(s), u(0, t)=\psi(t) .
\end{aligned}
$$

These last two equations are set in a Banach space so as to allow applications to integrodifferential equations such as

$$
\begin{aligned}
& \frac{\partial^{2}}{\partial s \partial t} u(s, t, z)= f(s, t, z)+H\left(z, u(s, t, z), \frac{\partial}{\partial s} u(s, t, z), \frac{\partial}{\partial t} u(s, t, z)\right) \\
&+\int_{0}^{1} K\left(z, r, u(s, t, r), \frac{\partial}{\partial s} u(s, t, r), \frac{\partial}{\partial t} u(s, t, r)\right) d r \\
& u(s, 0, z)=\sigma(s, z), u(0, t, z)=\tau(t, z) .
\end{aligned}
$$

Presented in part to the Society, November 19, 1971 under the title Existence on prescribed rectangles for a hyperbolic equation in a Banach space; received by the editors January 18, 1972.

AMS (MOS) subject classifications (1970). Primary 34D10, 45D05, 47H10; Secondary 35L60, 45K05, 34G05.

Key words and phrases. Fixed point theorems, perturbed differential equations, multivariable Volterra integral equations, hyperbolic equations in function spaces, hyperbolic integrodifferential equations. 
I. Introduction. In studying functional differential equations of the form

$$
u^{\prime}(t)=f(t)+A(t, u(t))+G[u](t), \quad u(0)=p,
$$

one frequently considers the problem as a perturbation of the ordinary differential equation

$$
v^{\prime}(t)=g(t)+A(t, v(t)), \quad v(0)=q .
$$

If $T$ is a function having the property that $v=T[q, g]$ (where $v, q$, and $g$ are as in (2)), then any solution $u$ of (1) satisfies $u=T[p, f+G[u]]$, i.e., $u$ is a fixed point of the mapping described by $w \rightarrow T[p, f+G[w]]$. This point of view has been exploited by many authors, both for perturbed differential equations and for perturbed Volterra integral equations (see, for example, R. K. Miller, J. A. Nohel, and J.S. W. Wong [13], [14], Miller [11], [12], M. Z. Nashed and Wong [16], and S. I. Grossman and Miller [4]).

In $\$$ II we shall develop a fixed point theorem designed to include not only (1) but also other applications. The results concerning (1) will be applied to Fredholm integrodifferential equations of the forms

$$
z^{\prime}(t)=f(t)+\phi(t, z(t))+\int_{0}^{\infty} \alpha(t, s) \omega(z(s)) d s, \quad z(0)=p
$$

and

$$
z^{\prime}(t)=f(t)+\phi(t, z(t))+\int_{0}^{\infty} \kappa(t-s) \omega(z(s)) d s, \quad z(0)=p .
$$

In $\S \mathrm{IV}$ we shall obtain existence and uniqueness results on prescribed rectangles for integral equations of the form

$$
\begin{aligned}
& u_{1}(s, t)=g_{1}(s, t)+\int_{0}^{s} \int_{0}^{t} F\left[u_{1}(x, y), u_{2}(x, y), u_{3}(x, y)\right] d y d x, \\
& u_{2}(s, t)=g_{2}(s, t)+\int_{0}^{t} F\left[u_{1}(s, y), u_{2}(s, y), u_{3}(s, y)\right] d y, \\
& u_{3}(s, t)=g_{3}(s, t)+\int_{0}^{s} F\left[u_{1}(x, t), u_{2}(x, t), u_{3}(x, t)\right] d x,
\end{aligned}
$$

and, as corollary to this, differential equations of the form

$$
\begin{aligned}
\frac{\partial^{2}}{\partial s \partial t} u(s, t)=f(s, t)+F\left[u(s, t), \frac{\partial}{\partial s} u(s, t), \frac{\partial}{\partial t} u(s, t)\right], & \\
u(s, 0) & =\phi(s), u(0, t)=\psi(t) .
\end{aligned}
$$

Such integral and differential equations have been studied under a variety of hypotheses by W. Walter [17], [18], [19] in finite-dimensional spaces. Our primary assumption will be the continuous Fréchet differentiability of the function $F$ from the triple product of a Banach space into that space. The technique here is similar to the linearization technique developed by Miller [12]. The corresponding 
ordinary differential equation has been studied by J. Dieudonné [3, Chapter 10, \$]. It will be indicated at the end of $\$ I V$ that the Banach space setting of (6) permits the realization of information about equations such as

$$
\begin{array}{r}
\frac{\partial^{2}}{\partial s \partial t} u(s, t, z)=f(s, t, z)+H\left(z, u(s, t, z), \frac{\partial}{\partial s} u(s, t, z), \frac{\partial}{\partial t} u(s, t, z)\right) \\
+\int_{0}^{1} K\left(z, r, u(s, t, r), \frac{\partial}{\partial s} u(s, t, r), \frac{\partial}{\partial t} u(s, t, r)\right) d r, \\
u(s, 0, z)=\sigma(s, z), u(0, t, z)=\tau(t, z) .
\end{array}
$$

II. A fixed point theorem. In this section we shall take $X_{1}$ and $X_{2}$ to be Banach spaces with norms $\pi_{1}$ and $\pi_{2}$ respectively. If $d$ is a positive number then $\mathcal{S}_{1}(d)$ will be that subset of $X_{1}$ to which $p$ belongs only in case $\pi_{1}[p] \leq d$, and $\mathcal{S}_{2}(d)$ will be the analogous subset of $X_{2}$. Let $T$ be a function from $X_{1} \times$ $X_{2}$ to $X_{2}$, let $G$ be a function from $X_{2}$ to $X_{2}$, and suppose that $T[0,0]=G[0]$ $=0$. Let $\beta$ be a number and suppose that

$$
\varkappa_{2}[T[p, f]-T[q, g]] \leq \beta \varkappa_{1}[p-q]+\beta \varkappa_{2}[f-g]
$$

whenever $(p, q, f, g)$ is in $X_{1} \times X_{1} \times X_{2} \times X_{2}$. Suppose that $G$ is of bigher order in the sense that if $\epsilon$ is a positive number then there is a positive number $\delta$ such that

$$
\pi_{2}[G[f]-G[g]] \leq \epsilon \pi_{2}[f-g]
$$

whenever $(f, g)$ is in $\mathcal{S}_{2}(\delta) \times \mathcal{S}_{2}(\delta)$.

Theorem 1. There are positive numbers $\eta$ and $\sigma$ such that, if $(p, f)$ is in $\mathcal{S}_{1}(\eta) \times \mathcal{S}_{2}(\eta)$, then there is exactly one member $u$ of $\mathcal{S}_{2}(\sigma)$ sucb that $u=$ $T[p, f+G[u]]$. Furthermore, if $R$ is that function from $\mathcal{S}_{1}(\eta) \times \mathcal{S}_{2}(\eta)$ to $\mathcal{S}_{2}(\sigma)$ baving the property that $R[p, f]=T[p, f+G[R[p, f]]]$ whenever $(p, f)$ is in $\mathcal{S}_{1}(\eta) \times \mathcal{S}_{2}(\eta)$, then $R$ is continuous.

Proof. If $\beta=0$, the theorem is trivial, so assume that $\beta>0$. Find a positive number $\sigma$ such that $\pi_{2}[G[f]-G[g]] \leq(1 / 4 \beta) \pi_{2}[f-g]$ whenever $(f, g)$ is in $\mathcal{S}_{2}(\sigma) \times \mathcal{S}_{2}(\sigma)$. Let $\eta=\sigma / 4 \beta$, and let $(p, f)$ be in $\mathcal{S}_{1}(\eta) \times \mathcal{S}_{2}(\eta)$. Let $K$ be the function from $\mathcal{S}_{2}(\sigma)$ to $X_{2}$ given by $K[g]=T[p, f+G[g]]$. Suppose that $g$ is in $\mathcal{S}_{2}(\sigma)$. Now

$$
\begin{aligned}
\Re_{2}[K[g]] & \leq \beta \varkappa_{1}[p]+\beta \varkappa_{2}[f]+\beta \varkappa_{2}[G[g]] \\
& \leq \sigma / 4+\sigma / 4+\sigma / 4<\sigma,
\end{aligned}
$$

so $K$ maps $\mathcal{S}_{2}(\sigma)$ into $\mathcal{S}_{2}(\sigma)$. Suppose that $(g, b)$ is in $\mathcal{S}_{2}(\sigma) \times \mathcal{S}_{2}(\sigma)$. Now 


$$
\Re_{2}[K[g]-K[b]] \leq \beta \Re_{2}[G[g]-G[b]] \leq(1 / 4) \Re_{2}[g-b] .
$$

Thus $K$ is a contraction, and there is exactly one member $u$ of $\delta_{2}(\sigma)$ such that $u=T[p, f+G[u]]$. This continuity of $R$ is now easy to see, and the proof is complete.

Note that the coup de grâce in the proof of Theorem 1 was administered with the contraction principle. With this in mind it is clear that Theorem 1 could be generalized along the lines of more sophisticated fixed point theorems (see, for example, M. A. Krasnoselskii [5], Nashed and Wong [16], or the recent work in linear topological spaces of G. L. Cain, Jr., and Nashed [1]). For our present purposes Theorem 1 as stated will suffice. Note also that $\eta$ and $\sigma$ were chosen so as to ensure that $\pi_{2}[u] \leq(3 \sigma) / 4$. This is not necessary for the success of Theorem 1, but will be useful below, in Theorem 4 .

A useful special case of Theorem 1 is the case in which $T$ is simply a function from $X_{2}$ to $X_{2}, T[0]=0$, and

$$
\gamma_{2}[T[f]-T[g]] \leq \beta \Re_{2}[f-g]
$$

whenever $(f, g)$ is in $X_{2} \times X_{2}$. In this case, let $T^{*}$ from $X_{1} \times X_{2}$ to $X_{2}$ be given by $T^{*}[p, f]=T[f]$, and Theorem 1 applies. Also, in this case, it is clear that $\pi_{2}[u] \leq \sigma / 2$.

III. A functional differential equation. Let $Y$ be a Banach space with norm $\mid$, and let $R^{+}$be the set of all nonnegative real numbers. Let $\mathfrak{B C}$ be the set to which $f$ belongs only in case $f$ is a bounded continuous funtcion from $R^{+}$to $Y$, and, if $f$ is in $\mathfrak{B C}$, let $J[f]$ be the least number $c$ such that $|f(s)| \leq c$ whenever $s$ is in $R^{+}$. Let $A$ be a continuous function $R^{+} \times Y$ to $Y$, and suppose that

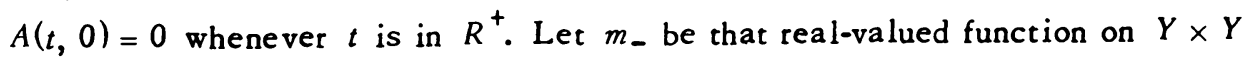
described by

$$
m_{-}[p, q]=\lim _{\delta \rightarrow 0_{-}}(1 / \delta)(|p+\delta q|-|p|) .
$$

Let $\rho$ be a continuous real-valued function on $R^{+}$, and let $G$ be a function from $B C$ to $B C$. We will find conditions (C1), (C2), and (C3) to be useful.

(C1) There is a number $\beta$ such that

$$
\exp \left[\int_{0}^{t} \rho(r) d r\right]+\int_{0}^{t} \exp \left[\int_{s}^{t} \rho(r) d r\right] d s \leq \beta
$$

whenever $t$ is in $R^{+}$.

(C2) If $(t, p, q)$ is in $R^{+} \times Y \times Y$ then

$$
m_{-}[p-q, A(t, p)-A(t, q)] \leq \rho(t)|p-q| .
$$


(C3) $G[0]=0$, and $G$ is of higher order.

Theorem 2. Suppose that eacb of (C1), (C2), and (C3) is true. Then there are positive numbers $\eta$ and $\sigma$ sucb that if $(p, f)$ is in $Y \times B \mathcal{C}$ and $|p| \leq \eta$ and $J[f] \leq \eta$ then there is exactly one member $u$ of $B C$ such that $J[u] \leq \sigma$ and such that (1) is true whenever $t$ is a positive number.

Equations similar to (2) have been studied extensively by R. H. Martin, Jr. [9], [10], [11] under a variety of hypotheses related to condition (C2). It follows from [7, Theorem 5] that (C2) implies (2) can be solved locally uniquely, and the addition of (C1) ensures that solutions, as far as they can be continued, are bounded. This author and Martin [8] have extended this to show that (C2) implies global existence for (2). In particular, if both (C1) and (C2) hold, it is then the case that if $(q, g)$ is in $Y \times \mathfrak{B C}$ then there is exactly one member $v$ of $\mathbb{B C}$ such that (2) is true whenever $t$ is a positive number. Our definition of higher order is the same as that of Grossman and Miller [4, Definition 2], and Theorem 2 is related to, but independent of, [4, Theorem 4]. Note that if $f$ is a function from a subset of $R^{+}$to $Y$, if $c$ is in the domain of $f$, if $f_{-}^{\prime}(c)$ (the left derivative of $f$ at c) exists, and if $Q$ is given on the domain of $f$ by $Q(t)=|f(t)|$, then $Q_{-}^{\prime}(c)$ exists and $Q_{-}^{\prime}(c)=m_{-}\left[f(c), f_{-}^{\prime}(c)\right]$ (compare [2, p. 3]). Note also [7, Lemma 6] that if $(p, q, r)$ is in $Y \times Y \times Y$ then $m_{-}[p, q+r] \leq m_{-}[p, q]+|r|$.

Indication of proof of Theorem 2. Let $T$ be that function from $Y \times \mathfrak{B C}$ to $B \mathcal{C}$ having the property that if $(q, g, v)$ is in $Y \times \mathfrak{B C} \times \mathfrak{B C}$ then $v=T[q, g]$ only in case $v^{\prime}(t)$ exists and (2) is true whenever $t$ is a positive number. Let $\beta$ be as in (C1). In light of Theorem 1 , it suffices to show that

$$
J[T[p, f]-T[q, g]] \leq \beta|p-q|+\beta J[f-g]
$$

whenever $(p, q, f, g)$ is in $Y \times Y \times \mathfrak{B C} \times \mathfrak{B C}$. Let $(p, q, f, g)$ be in $Y \times Y \times \mathfrak{B C}$ $\times \mathfrak{B C}$, let $u=T[p, f]$, let $v=T[q, g]$, and let $P$ be given on $R^{+}$by $P(t)=$ $|u(t)-v(t)|$. Now, if $t$ is a positive number,

$$
\begin{aligned}
P_{-}^{\prime}(t) & =m_{-}\left[u(t)-v(t), u^{\prime}(t)-v^{\prime}(t)\right] \\
& =m_{-}[u(t)-v(t), A(t, u(t))-A(t, v(t))+f(t)-g(t)] \\
& \leq \rho(t) P(t)+|f(t)-g(t)| \leq \rho(t) P(t)+J[f-g] .
\end{aligned}
$$

Hence $[6$, Theorem 1.4 .1 , p. 15],

$$
\begin{aligned}
|u(t)-v(t)| & =P(t) \\
& \leq|p-q| \exp \left[\int_{0}^{t} \rho(r) d r\right]+J[f-g] \int_{0}^{t} \exp \left[\int_{s}^{t} \rho(r) d r\right] d s \\
& \leq \beta|p-q|+\beta J[f-g]
\end{aligned}
$$


whenever $t$ is in $R^{+}$. Thus $J[u-v] \leq \beta|p-q|+\beta J[f-g]$, and the proof is complete.

We shall apply Theorem 2 to a Fredholm integrodifferential equation. For the remainder of this section, let $Y$ be the set of all real numbers, normed by absolute value. Let $\phi$ be a continuous function from $R^{+} \times Y$ to $Y$, suppose that $\phi(t, 0)$ $=0$ whenever $t$ is in $R^{+}$, and suppose that if $t$ is in $R^{+}$then the function from $Y$ to $Y$ described by $s \rightarrow(\phi(t, s)-s \rho(t))$ is nonincreasing. Now (C2) holds, for $\phi$. Suppose that $(\mathrm{Cl})$ is true. Let $\alpha$ be a continuous function from $R^{+} \times R^{+}$ to $Y$, let $d$ be a positive number, and suppose that $\int_{0}^{\infty}|\alpha(t, s)| d s \leq d$ whenever $t$ is in $R^{+}$. Suppose also that if $\left\{t_{k}\right\}_{k=0}^{\infty}$ is an $R^{+}$-valued sequence with limit $t_{0}$ then $\lim _{k \rightarrow \infty} \int_{0}^{\infty}\left|\alpha\left(t_{0}, s\right)-\alpha\left(t_{k}, s\right)\right| d s=0$. Suppose that $\omega$ is a continuous function from $Y$ to $Y$, suppose that there is an open neighborhood about 0 on which $\omega$ is continuously differentiable, and suppose that $\omega(0)=\omega^{\prime}(0)=0$.

Theorem 3. There are positive numbers $\eta$ and $\sigma$ such that, if $(p, f)$ is in $Y \times \mathfrak{B C}$ and $|p| \leq \eta$ and $J[f] \leq \eta$, then there is exactly one member $z$ of $\mathfrak{B C}$ such that $J[z] \leq \sigma$ and such that (3) is true whenever $t$ is a positive number.

Corollary 1. Suppose that $\kappa$ is a continuous function from $Y$ to $Y$, and suppose that $\int_{-\infty}^{\infty}|\kappa(s)| d s$ exists. Then the conclusions of Theorem 3 are true with respect to (4).

Corollary 1 follows immediately from Theorem 3, and we shall not exhibit a proof for it here.

Proof of Theorem 3. Let $G$ from $\mathcal{B C}$ to $\mathcal{B C}$ be given by

$$
G[b](t)=\int_{0}^{\infty} a(t, s) \omega(b(s)) d s .
$$

In light of Theorem 2, it suffices to show that $G$ is of higher order. Let $\epsilon$ be a positive number. Let $\delta$ be a positive number such that if $t$ is in $[-\delta, \delta]$ then $\omega^{\prime}(t)$ exists and is in $[-\epsilon / d, \epsilon / d]$. Now $|\omega(t)-\omega(s)| \leq(\epsilon / d)|t-s|$ whenever each of $s$ and $t$ is in $[-\delta, \delta]$. Suppose that $(f, g)$ is in $\mathfrak{B C} \times \mathfrak{B C}$ and $J[f] \leq \delta$ and $J[g] \leq \delta$. Now, if $t$ is in $R^{+}$,

$$
\begin{aligned}
\left|\int_{0}^{\infty} \alpha(t, s) \omega(f(s)) d s-\int_{0}^{\infty} \alpha(t, s) \omega(g(s)) d s\right| \\
\quad \leq \int_{0}^{\infty}|\alpha(t, s)||\omega(f(s))-\omega(g(s))| d s \\
\leq(\epsilon / d) J[f-g] \int_{0}^{\infty}|\alpha(t, s)| d s \leq \epsilon J[f-g],
\end{aligned}
$$

and the proof is complete. 
IV. A Volterra integral equation. In this section we shall treat the Volterra integral equation of the introduction. Again, let $Y$ be a Banach space with norm 11. Let $Y^{3}$ denote the product $Y \times Y \times Y$ with norm $N$ given by $N\left[\left(p_{1}, p_{2}, p_{3}\right)\right]=$ $\max \left\{\left|p_{1}\right|,\left|p_{2}\right|,\left|p_{3}\right|\right\}$. Suppose that $b$ is a positive number, and let $C$ be the set to which $g$ belongs only in case $g$ is a continuous function from $[0, b] \times[0, b]$ to $Y$. If $g$ is in $\mathcal{C}$ let $J[g]$ be the least number $c$ such that $|g(s, t)| \leq c$ whenever $(s, t)$ is in $[0, b] \times[0, b]$. Let $F$ be a continuous function from $Y^{3}$ to $Y$, suppose that $F[(0,0,0)]=0$, let $D$ be an open set in $Y^{3}$ containing $(0,0,0)$, and suppose that $F$ is continuously Fréchet differentiable on $D$ (we mean Fréchet derivative in the sense of $[3$, p. 149]).

Theorem 4. There is a positive number $\eta$ sucb that, if $\left(g_{1}, g_{2}, g_{3}\right)$ is in $\mathcal{C} \times \mathcal{C} \times \mathcal{C}$ and $J\left[g_{i}\right] \leq \eta$ whenever $i$ is in $\{1,2,3\}$, then there is exactly one member $\left(u_{1}, u_{2}, u_{3}\right)$ of $\mathcal{C} \times C_{\times} \mathcal{C}$ such that equations (5) are true whenever $(s, t)$ is in $[0, b] \times[0, b]$.

Corollary 2. There is a positive number $\eta$ such that if eacb of (i) and (ii) is true then (iii) is true.

(i) Each of $\phi$ and $\psi$ is a continuously differentiable function from $[0, b]$ to $Y, p$ is in $Y, \phi(0)=\psi(0)=p,|\phi(s)+\psi(t)-p| \leq \eta / 2$ whenever $(s, t)$ is in $[0, b] \times[0, b],\left|\phi^{\prime}(s)\right| \leq \eta / 2$ whenever $s$ is in $[0, b]$, and $\left|\psi^{\prime}(t)\right| \leq \eta / 2$ whenever $t$ is in $[0, b]$.

(ii) $f$ is a continuous function from $[0, b] \times[0, b]$ to $Y$ baving the property that if $(s, t)$ is in $[0, b] \times[0, b]$ then eacb of $\int_{0}^{s} \int_{0}^{t} f(x, y) d y d x, \int_{0}^{s} f(x, t) d x$, and $\int_{0}^{t} f(s, y) d y$ bas norm not exceeding $\eta / 2$.

(iii) There is exactly one continuously differentiable function $u$ from $[0, b] \times[0, b]$ to $Y$ such that $u(s, 0)=\phi(s)$ and $u(0, t)=\psi(t)$ whenever $(s, t)$ is in $[0, b] \times[0, b]$ and sucb that

$$
\frac{\partial^{2}}{\partial s \partial t} u(s, t)=f(s, t)+F\left[\left(u(s, t), \frac{\partial}{\partial s} u(s, t), \frac{\partial}{\partial t} u(s, t)\right)\right]
$$

whenever $(s, t)$ is in $(0, b) \times(0, b)$.

First we comment on how Corollary 2 follows from Theorem 4, and then we prove Theorem 4. Suppose that $\phi, \psi, p$, and $f$ are as in (i) and (ii) of Corollary 2.

Let $\left(g_{1}, g_{2}, g_{3}\right)$ in $\mathcal{C} \times \mathcal{C} \mathcal{C}_{\text {be given by }}$

$$
\begin{aligned}
& g_{1}(s, t)=\phi(s)+\psi(t)-p+\int_{0}^{s} \int_{0}^{t} f(x, y) d y d x, \\
& g_{2}(s, t)=\phi^{\prime}(s)+\int_{0}^{t} f(s, y) d y, g_{3}(s, t)=\psi^{\prime}(t)+\int_{0}^{s} f(x, t) d x .
\end{aligned}
$$


Now $J\left[g_{i}\right] \leq \eta$ whenever $i$ is in $\{1,2,3\}$, so Theorem 4 applies. Let $\left(u_{1}, u_{2}, u_{3}\right)$ be as in the conclusion of Theorem 4. Now clearly $u_{1}(s, 0)=\phi(s)$ and $u_{1}(0, t)=$ $\psi(t)$ whenever $(s, t)$ is in $[0, b] \times[0, b]$. Also,

$$
\begin{aligned}
\frac{\partial}{\partial s} u_{1}(s, t) & =\phi^{\prime}(s)+\int_{0}^{t} f(s, y) d y+\int_{0}^{t} F\left[\left(u_{1}(s, y), u_{2}(s, y), u_{3}(s, y)\right)\right] d y \\
& =u_{2}(s, t)
\end{aligned}
$$

and, similarly, $(\partial / \partial t) u_{1}(s, t)=u_{3}(s, t)$ whenever $(s, t)$ is in $(0, b) \times(0, b)$. Thus

$$
\begin{aligned}
\frac{\partial^{2}}{\partial s \partial t} u_{1}(s, t) & =f(s, t)+F\left[\left(u_{1}(s, t), u_{2}(s, t), u_{3}(s, t)\right)\right] \\
& =f(s, t)+F\left[\left(u_{1}(s, t), \frac{\partial}{\partial s} u_{1}(s, t), \frac{\partial}{\partial t} u_{1}(s, t)\right)\right]
\end{aligned}
$$

whenever $(s, t)$ is in $(0, b) \times(0, b)$. If, on the other hand, $v$ is another function satisfying (iii) of Corollary 2 , then the triple $(v, \partial v / \partial s, \partial v / \partial t)$ satisfies Theorem 4 with the above selected $\left(g_{1}, g_{2}, g_{3}\right)$, so $v=u_{1}$. It is now clear that Theorem 4 includes Corollary 2.

Proof of Theorem 4. Let $A$ be the Fréchet derivative of $F$ at $(0,0,0)$, i.e., $A$ is a continuous linear function from $Y^{3}$ to $Y$ and

$$
\left|A\left[\left(p_{1}, p_{2}, p_{3}\right)\right]-F\left[\left(p_{1}, p_{2}, p_{3}\right)\right]\right| / N\left[\left(p_{1}, p_{2}, p_{3}\right)\right] \rightarrow 0
$$

as $N\left[\left(p_{1}, p_{2}, p_{3}\right)\right] \rightarrow 0$. Let $\mathcal{C}^{3}$ denote the product $\mathcal{C} \times \mathcal{C} \times \mathcal{C}$ with norm $J_{3}$ given by $J_{3}\left[\left(g_{1}, g_{2}, g_{3}\right)\right]=\max \left\{J\left[g_{1}\right], J\left[g_{2}\right], J\left[g_{3}\right]\right\}$. Let $m$ be a number such that $m \geq 1$ and such that $\left|A\left[\left(p_{1}, p_{2}, p_{3}\right)\right]\right| \leq m N\left[\left(p_{1}, p_{2}, p_{3}\right)\right]$ whenever $\left(p_{1}, p_{2}, p_{3}\right)$ is in $Y^{3}$. Let $R$ be that continuous linear function from $\mathcal{C}^{3} \times \mathcal{C}^{3}$ to $\mathcal{C}^{3}$ having the property that $\left(v_{1}, v_{2}, v_{3}\right)=R\left[\left(g_{1}, g_{2}, g_{3}\right),\left(w_{1}, w_{2}, w_{3}\right)\right]$ only in case

$$
\begin{aligned}
& v_{1}(s, t)=g_{1}(s, t)+\int_{0}^{s} \int_{0}^{t} A\left[\left(w_{1}(x, y), w_{2}(x, y), w_{3}(x, y)\right)\right] d y d x, \\
& v_{2}(s, t)=g_{2}(s, t)+\int_{0}^{t} A\left[\left(w_{1}(s, y), w_{2}(s, y), w_{3}(s, y)\right)\right] d y,
\end{aligned}
$$

and

$$
v_{3}(s, t)=g_{3}(s, t)+\int_{0}^{t} A\left[\left(w_{1}(x, t), w_{2}(x, t), w_{3}(x, t)\right)\right] d x
$$

whenever $(s, t)$ is in $[0, b] \times[0, b]$. Now let $K$ be the norm on $\mathcal{C}^{3}$ having the property that if $\left(g_{1}, g_{2}, g_{3}\right)$ is in $C^{3}$ then $K\left[\left(g_{1}, g_{2}, g_{3}\right)\right]$ is the least number $c$ such that $e^{-2 m(s+t)} N\left[\left(g_{1}(s, t), g_{2}(s, t), g_{3}(s, t)\right)\right] \leq c$ whenever $(s, t)$ is in $[0, b] \times[0, b]$. Now $K$ is clearly an equivalent norm to $J_{3}$, and, since $m \geq 1$, a computation almost identical to the computation in $[18$, p. 11] shows that 


$$
\begin{array}{r}
\left.K\left[R\left[\left(g_{1}, g_{2}, g_{3}\right),\left(v_{1}, v_{2}, v_{3}\right)\right]-R\left[\left(g_{1}, g_{2}, g_{3}\right), w_{1}, w_{2}, w_{3}\right)\right]\right] \\
\leq(1 / 2) K\left[\left(v_{1}, v_{2}, v_{3}\right)-\left(w_{1}, w_{2}, w_{3}\right)\right]
\end{array}
$$

whenever $\left(\left(g_{1}, g_{2}, g_{3}\right),\left(v_{1}, v_{2}, v_{3}\right),\left(w_{1}, w_{2}, w_{3}\right)\right)$ is in $\mathcal{C}^{3} \times \mathcal{C}^{3} \times \mathcal{C}^{3}$. Hence there is a continuous linear function $T$ from $\mathcal{C}^{3}$ to $\mathcal{C}^{3}$ such that $T\left[\left(g_{1}, g_{2}, g_{3}\right)\right]$ $=R\left[\left(g_{1}, g_{2}, g_{3}\right), T\left[\left(g_{1}, g_{2}, g_{3}\right)\right]\right]$ whenever $\left(g_{1}, g_{2}, g_{3}\right)$ is in $\mathcal{C}^{3}$. Now, since $T$ is linear and continuous, there is a number $\beta$ such that $J_{3}\left[T\left[\left(g_{1}, g_{2}, g_{3}\right)\right]\right] \leq$ $\beta J_{3}\left[\left(g_{1}, g_{2}, g_{3}\right)\right]$ whenever $\left(g_{1}, g_{2}, g_{3}\right)$ is in $\mathcal{C}^{3}$. Choose $\beta>1$.

Let $G$ be that function from $\mathcal{C}^{3}$ to $\mathcal{C}^{3}$ such that if $\left(\left(v_{1}, v_{2}, v_{3}\right),\left(w_{1}, w_{2}, w_{3}\right)\right)$ is in $\mathcal{C}^{3} \times \mathcal{C}^{3}$ then $\left(v_{1}, v_{2}, v_{3}\right)=G\left[\left(w_{1}, w_{2}, w_{3}\right)\right]$ only in case

$$
\begin{aligned}
& v_{1}(s, t)=\int_{0}^{s} \int_{0}^{t}[F[\left(w_{1}(x, y), w_{2}(x, y), w_{3}(x, y)\right] \\
&-A\left[\left(w_{1}(x, y), w_{2}(x, y), w_{3}(x, y)\right]\right] d y d x, \\
& v_{2}(s, t)=\int_{0}^{t}\left[F\left[\left(w_{1}(s, y), w_{2}(s, y), w_{3}(s, y)\right)\right]\right. \\
&\left.-A\left[\left(w_{1}(s, y), w_{2}(s, y), w_{3}(s, y)\right)\right]\right] d y,
\end{aligned}
$$

and

$$
\begin{aligned}
& v_{3}(s, t)=\int_{0}^{s}\left[F\left[\left(w_{1}(x, t), w_{2}(x, t), w_{3}(x, t)\right)\right]\right. \\
&\left.-A\left[\left(w_{1}(x, t), w_{2}(x, t), w_{3}(x, t)\right)\right]\right] d x
\end{aligned}
$$

whenever $(s, t)$ is in $[0, b] \times[0, b]$. Clearly now $G[(0,0,0)]=(0,0,0)$. Suppose that $\epsilon$ is a positive number. Let $c=\max \left\{b, b^{2}\right\}$. If $\left(p_{1}, p_{2}, p_{3}\right)$ is in $D$, let $F^{\prime}\left(p_{1}, p_{2}, p_{3}\right)$ denote the Fréchet derivative of $F$ at $\left(p_{1}, p_{2}, p_{3}\right)$. Now, by continuity of $F^{\prime}$, there is a positive number $\delta$ such that if $\left(p_{1}, p_{2}, p_{3}\right)$ is in $Y^{3}$ and $N\left[\left(p_{1}, p_{2}, p_{3}\right)\right] \leq \delta$ then $\left(p_{1}, p_{2}, p_{3}\right)$ is in $D$ and the operator norm of $F^{\prime}\left(p_{1}, p_{2}, p_{3}\right)-A$ does not exceed $\epsilon / c$. Hence [3, (8.5.4)],

$$
\begin{aligned}
& \mid\left[F\left[\left(p_{1}, p_{2}, p_{3}\right)\right]-\right.\left.A\left[\left(p_{1}, p_{2}, p_{3}\right)\right]\right] \\
&-\left[F\left[\left(q_{1}, q_{2}, q_{3}\right)\right]-A\left[\left(q_{1}, q_{2}, q_{3}\right)\right]\right] \mid \\
& \leq(\epsilon / c) N\left[\left(p_{1}, p_{2}, p_{3}\right)-\left(q_{1}, q_{2}, q_{3}\right)\right]
\end{aligned}
$$

whenever each of $\left(p_{1}, p_{2}, p_{3}\right)$ and $\left(q_{1}, q_{2}, q_{3}\right)$ is in $Y^{3}$ and $N\left[\left(p_{1}, q_{2}, q_{3}\right)\right] \leq \delta$ and $N\left[\left(q_{1}, q_{2}, q_{3}\right)\right] \leq \delta$. It is now clear that

$$
J_{3}\left[G\left[\left(v_{1}, v_{2}, v_{3}\right)\right]-G\left[\left(w_{1}, w_{2}, w_{3}\right)\right]\right] \leq \epsilon J_{3}\left[\left(v_{1}, v_{2}, v_{3}\right)-\left(w_{1}, w_{2}, w_{3}\right)\right]
$$

whenever each of $\left(v_{1}, v_{2}, v_{3}\right)$ and $\left(w_{1}, w_{2}, w_{3}\right)$ is in $\mathcal{C}^{3}$ and $J_{3}\left[\left(v_{1}, v_{2}, v_{3}\right)\right] \leq \delta$ and $J_{3}\left[\left(w_{1}, w_{2}, w_{3}\right)\right] \leq \delta$. Thus $G$ is of higher order. Choose $\eta$ and $\sigma$ as in the 
proof of Theorem 1. Suppose that $\left(g_{1}, g_{2}, g_{3}\right)$ is in $C^{3}$ and $J_{3}\left[\left(g_{1}, g_{2}, g_{3}\right)\right] \leq \eta$. Now there is exactly one member $\left(u_{1}, u_{2}, u_{3}\right)$ of $\mathcal{C}^{3}$ such that $J_{3}\left[\left(u_{1}, u_{2}, u_{3}\right)\right] \leq \sigma$ and such that

$$
\left(u_{1}, u_{2}, u_{3}\right)=T\left[\left(g_{1}, g_{2}, g_{3}\right)+G\left[\left(u_{1}, u_{2}, u_{3}\right)\right]\right]
$$

This member $\left(u_{1}, u_{2}, u_{3}\right)$ of $\mathcal{C}^{3}$ clearly satisfies equations (5). The proof, however, is not yet complete since we as yet have uniqueness only with respect to a subset of $\mathcal{C}^{3}$.

Suppose that $\left(\left(g_{1}, g_{2}, g_{3}\right),\left(v_{1}, v_{2}, v_{3}\right)\right)$ is in $\mathcal{C}^{3} \times \mathcal{C}^{3}, J_{3}\left[\left(g_{1}, g_{2}, g_{3}\right)\right] \leq \delta$, and $\left(v_{1}, v_{2}, v_{3}\right)=T\left[\left(g_{1}, g_{2}, g_{3}\right)+G\left[\left(v_{1}, v_{2}, v_{3}\right)\right]\right]$. To complete the proof it suffices to show that $J_{3}\left[\left(v_{1}, v_{2}, v_{3}\right)\right]<\sigma$. Suppose that there is $(s, t)$ in $[0, b] \times$ $[0, b]$ and $i$ in $\{1,2,3\}$ such that $\left|v_{i}(s, t)\right|=\sigma$. Let $d$ be the largest member of $[0, b]$ such that $N\left[\left(v_{1}(s, t), v_{2}(s, t), v_{3}(s, t)\right)\right]<\sigma$ whenever $(s, t)$ is in $[0, d) \times$ $[0, d)$. Now $\left|v_{i}(0,0)\right|=\left|g_{i}(0,0)\right| \leq \eta=\sigma / 4 \beta<\sigma$ whenever $i$ is in $\{1,2,3\}$ so $d$ is positive (recall that we chose $\beta>1$ ). Let $\mathcal{C}^{*}$ be the collection of all continuous functions from $[0, d] \times[0, d]$ to $Y$, and define $\mathcal{C}^{* 3}, T^{*}, J_{3}^{*}$, and $G^{*}$ analogously. Now $J_{3}^{*}\left[T^{*}\left[\left(b_{1}^{*}, b_{2}^{*}, b_{3}^{*}\right)\right]\right] \leq \beta J_{3}^{*}\left[\left(b_{1}^{*}, b_{2}^{*}, b_{3}^{*}\right)\right]$ whenever $\left(b_{1}^{*}, b_{2}^{*}, b_{3}^{*}\right)$ is in $\mathcal{C}^{* 3}$, and $J_{3}^{*}\left[G^{*}\left[\left(b_{1}^{*}, b_{2}^{*}, b_{3}^{*}\right)\right]\right] \leq(1 / 4 \beta) J_{3}^{*}\left[\left(b_{1}^{*}, b_{2}^{*}, b_{3}^{*}\right)\right]$ whenever $\left(b_{1}^{*}, b_{2}^{*}, b_{3}^{*}\right)$ is in $C^{* 3}$ and $J_{3}^{*}\left[\left(b_{1}^{*}, b_{2}^{*}, b_{3}^{*}\right)\right] \leq \sigma$. If $i$ is in $\{1,2,3\}$, let $g_{i}^{*}$ and $v_{i}^{*}$ be the restrictions of $g_{i}$ and $v_{i}$, respectively, to $[0, d] \times[0, d]$. Now

$$
\begin{aligned}
J_{3}^{*}\left[\left(v_{1}^{*}, v_{2}^{*}, v_{3}^{*}\right)\right] & =J_{3}^{*}\left[T^{*}\left[\left(g_{1}^{*}, g_{2}^{*}, g_{3}^{*}\right)+G^{*}\left[\left(v_{1}^{*}, v_{2}^{*}, v_{3}^{*}\right)\right]\right]\right] \\
& \leq J_{3}^{*}\left[\left(g_{1}^{*}, g_{2}^{*}, g_{3}^{*}\right)\right]+J_{3}^{*}\left[G^{*}\left[\left(v_{1}^{*}, v_{2}^{*}, v_{3}^{*}\right)\right]\right] \\
& \leq \sigma / 4+\sigma / 4=\sigma / 2 .
\end{aligned}
$$

Thus, if $i$ is in $\{1,2,3\}$, and $(s, t)$ is in $[0, d] \times[0, d]$,

$$
\begin{aligned}
\left|v_{i}(s, t)\right| & =\left|v_{i}^{*}(s, t)\right| \\
& \leq N\left[\left(v_{1}^{*}(s, t), v_{2}^{*}(s, t), v_{3}^{*}(s, t)\right] \leq J_{3}^{*}\left[\left(v_{1}^{*}, v_{2}^{*}, v_{3}^{*}\right)\right] \leq \sigma / 2 .\right.
\end{aligned}
$$

So we have a contradiction, and the proof of Theorem 4 is complete.

It should be noted (see, in particular, the work of W. Waiter [17], [18], [19]) that a wide class of partial differential initial value problems can be rephrased into the language of multivariable Volterra integral equations, and hence can be dealt with by the methods we used in Theorem 4 and Corollary 2. Note also, in Theorem 4, that $\left(u_{1}, u_{2}, u_{3}\right)$ "depends continuously" on $\left(g_{1}, g_{2}, g_{3}\right)$ and hence, in Corollary 2, $u, \partial u / \partial s$, and $\partial u / \partial t$ "depend continuously" on $\phi, \psi, \phi^{\prime}, \psi^{\prime}$, and $f$.

Corollary 2 can be used to solve some classes of integrodifferential equations which can be written as partial differential equations over an appropriate space 
of functions. Consider, for example, equation (7).

Let $R$ be the set of all real numbers, let $S$ be the interval $[0,1]$, and let $Y$ be the set of all continuous functions from $S$ to $R$, normed with the supremum norm. Let $H$ be a continuous function from $S \times R \times R \times R$ to $R$, and let $K$ be a continuous function from $\mathrm{S} \times \mathrm{S} \times \mathrm{R} \times \mathrm{R} \times \mathrm{R}$ to $\mathrm{R}$. Let $\mathrm{H}_{2}, \mathrm{H}_{3}$, and $\mathrm{H}_{4}$ denote the partial derivatives of $H$ with respect to its second, third, and fourth positions respectively, and let $K_{3}, K_{4}$, and $K_{5}$ denote the partial derivatives of $K$ with respect to its third, fourth, and fifth positions respectively. We suppose that each of $\mathrm{H}_{2}, \mathrm{H}_{3}$, and $\mathrm{H}_{4}$ is continuous from $S \times R \times R \times R$ to $R$, and that each of $K_{3}, K_{4}$, and $K_{5}$ is continuous from $S \times S \times R \times R \times R$ to $R$. Suppose also that $H(z, 0,0,0)=0$ and $\int_{0}^{1} K(z, r, 0,0,0) d r=0$ whenever $z$ in in $S$. Let $F_{1}$ be given from $Y^{3}$ to $Y$ by

$$
F_{1}\left[\left(p_{1}, p_{2}, p_{3}\right)\right](z)=H\left(z, p_{1}(z), p_{2}(z), p_{3}(z)\right),
$$

and let $F_{2}$ be given from $Y^{3}$ to $Y$ by

$$
F_{2}\left[\left(p_{1}, p_{2}, p_{3}\right)\right](z)=\int_{0}^{1} K\left(z, r, p_{1}(r), p_{2}(r), p_{3}(r)\right) d r .
$$

Let $F=F_{1}+F_{2}$.

Suppose that $z$ is in $S$, and each of $x_{1}, x_{2}, x_{3}, c_{1}, c_{2}$, and $c_{3}$ is in $R$. Now the mean value theorem tells us that there are members $d_{1}, d_{2}$, and $d_{3}$ of $R$ such that $\left|d_{i}\right| \leq\left|c_{i}\right|$ whenever $i$ is in $\{1,2,3\}$ and such that

$$
\begin{aligned}
& \mid\left[H\left(z, x_{1}+c_{1}, x_{2}+c_{2}, x_{3}+c_{3}\right)-H\left(z, x_{1}, x_{2}, x_{3}\right)\right] \\
& \left.\quad-\mid c_{1} H_{2}\left(z, x_{1}, x_{2}, x_{3}\right)+c_{2} H_{3}\left(z, x_{1}, x_{2}, x_{3}\right)+c_{3} H_{4}\left(z, x_{1}, x_{2}, x_{3}\right)\right] \mid \\
& \leq \mid H\left(z, x_{1}+c_{1}, x_{2}+c_{2}, x_{3}+c_{3}\right)-H\left(z, x_{1}+c_{1}, x_{2}+c_{2}, x_{3}\right) \\
& \quad-c_{3} H_{4}\left(z, x_{1}, x_{2}, x_{3}\right) \mid \\
& +\mid H\left(z, x_{1}+c_{1}, x_{2}+c_{2}, x_{3}\right)-H\left(z, x_{1}+c_{1}, x_{2}, x_{3}\right) \\
& +\left|H\left(z, x_{1}+c_{1}, x_{2}, x_{3}\right)-H\left(z, x_{1}, x_{2}, x_{3}, x_{3}\right)-c_{1} H_{2}\left(z, x_{1}, x_{2}, x_{3}\right)\right| \\
& =\left|H_{4}\left(z, x_{1}+c_{1}, x_{2}+c_{2}, x_{3}+d_{3}\right)-H_{4}\left(z_{1}, x_{1}, x_{2}, x_{3}\right)\right|\left|c_{3}\right| \\
& +\left|H_{3}\left(z, x_{1}+c_{1}, x_{2}+d_{2}, x_{3}\right)-H_{3}\left(z, x_{1}, x_{2}, x_{3}\right)\right|\left|c_{2}\right| \\
& +\left|H_{2}\left(z, x_{1}+d_{1}, x_{2}, x_{3}\right)-H_{2}\left(z, x_{1}, x_{2}, x_{3}\right)\right|\left|c_{1}\right| .
\end{aligned}
$$

This computation, the observation that each of $\mathrm{H}_{2}, \mathrm{H}_{3}$, and $\mathrm{H}_{4}$ is uniformly continuous on compact subsets of its domain, and the observation that each member 
of $Y$ has range a compact subset of $R$, shows that $F_{1}$ is continuously Fréchet differentiable. Similarly, $F_{2}$ is continuously Fréchet differentiable, so $F$ is continuously Fréchet differentiable. Note that if $\left(p_{1}, p_{2}, p_{3}\right)$ is in $Y^{3}$, and $B$ is the Fréchet derivative of $F$ at $\left(p_{1}, p_{2}, p_{3}\right)$, then $B$ is given on $Y^{3}$ by

$$
\begin{aligned}
B\left[\left(q_{1}, q_{2}, q_{3}\right)\right](z) & =\sum_{k=1}^{3} H_{k+1}\left(z, p_{1}(z), p_{2}(z), p_{3}(z)\right) q_{k}(z) \\
& +\sum_{k=1}^{3} \int_{0}^{1} K_{k+2}\left(z, r, p_{1}(r), p_{2}(r), p_{3}(r)\right) q_{k}(r) d r .
\end{aligned}
$$

Clearly now (7) falls within the scope of Corollary 2. One final word here: Suppose that $b$ is a positive number and $g$ is a continuous function from $[0, b] \times$ $[0,1]$ to $R$. Suppose also that $g_{1}$, the partial derivative of $g$ with respect to its first position, is continuous from $[0, b] \times[0,1]$ to $R$. Let $b$ be the function from $[0, b]$ to $Y$ given by $b(t)=g(t$,$) . Now b$ is continuously differentiable and $b^{\prime}(t)=g_{1}(t$,$) whenever t$ is in $[0, b]$. This is well known and can be easily seen from a mean value theorem computation similar to the one above. Note that this shows how to restrict $\sigma$ and $\tau$ of (7) so as to satisfy (i) of Corollary 2.

\section{REFERENCES}

1. G. L. Cain, Jr. and M. Z. Nashed, Fixed points and stability for a sum of two operators in locally convex spaces, Pacific J. Math. 39 (1971), 581-592.

2. W. A. Coppel, Stability and asymptotic behavior of differential equations, Heath, Boston, Mass., 1965. MR 32 \#7875.

3. J. Dieudonné, Fondements.de l'analyse moderne, Pure and Appl. Math., vol. 10, Academic Press, New York and London, 1960. MR 22 \#11074.

4. S. I. Grossman and R. K. Miller, Perturbation theory for Volterra integrodifferential systems, J. Differential Equations 8 (1970), 457-474. MR 42 \#4988.

5. M. A. Krasnosel'skir, Two remarks on the method of successive approximations, Uspehi Mat. Nauk 10 (63) (1955), 123-127. (Russian) MR 16, 833.

6. V. Lakshmikantham and S. Leela, Differential and integral inequalities. Vol. 1, Academic Press, New York, 1969.

7. D. L. Lovelady, A functional differential equation in a Banach space, Funkcial. Ekvac. 14 (1971), 111-122.

8. D. L. Lovelady and R. H. Martin, Jr., A global existence theorem for a nonautonomous differential equation in a Banach space, Proc. Amer. Math. Soc. 35 (1972), 445-449.

9. R. H. Martin, Jr., The logarithmic derivative and equations of evolution in a Banach space, J. Math. Soc. Japan 22 (1970), 411-429.

10. - A global existence theorem for autonomous differential equations in a Banach space, Proc. Amer. Math. Soc. 26 (1970), 307-314. MR 41 \#8791.

11. Lyapunov functions and autonomous differential equations in a Banach space, Math. Systems Theory 7 (1973), 66-72. 
12. R. K. Miller, On the linearization of Volterra integral equations, J. Math. Anal. Appl. 23 (1968), 198-208. MR 37 \# 5635.

13. - Admissibility and nonlinear Volterra integral equations, Proc. Amer. Math. Soc. 25 (1970), 65-71. MR 41 \# 2324.

14. R. K. Miller, J. A. Nohel and J. S. W. Wong, A stability theorem for nonlinear mixed integral equations, J. Math. Anal. Appl. 25 (1969), 446-449. MR 38 \# 2556.

15. - Perturbations of Volterra integral equations, J. Math. Anal. Appl. 25 (1969), 676-691. MR 39 \#1920.

16. M. A. Nashed and J. S. W. Wong, Some variants of a fixed point theorem of Krasnoselskii and applications to nonlinear integral equations, J. Math. Mech. 18 (1969), 767-777. MR 38 \#6416.

17. W. Walter, Differential- und Integral-Ungleichungen und ihre Anwendung bei Abschätzungs - und Eindeutigkeitsproblem, Springer Tracts in Natural Philos., Band 2, Springer-Verlag, Berlin, 1964. MR 30 \#2302.

18. - Über sukzessive Approximation bei Volterra Integralgleichugen in mehreren Veränderlichen, Ann. Acad. Sci. Fenn. Ser. A I 345 (1965), pp. 32 . MR 34 \#1815.

19. - Differential and integral inequalities, Ergebnisse der Math. und ihrer Grenzgebiete, Band 55, Springer-Verlag, New York, 1970. MR 42 \#6391.

DEPARTMENT OF MATHEMATICS, UNIVERSITY OF SOUTH CAROLINA, COLUMBIA, SOUTH CAROLINA 29208

Current address: Department of Mathematics, Florida State University, Tallahassee, Florida 32306 